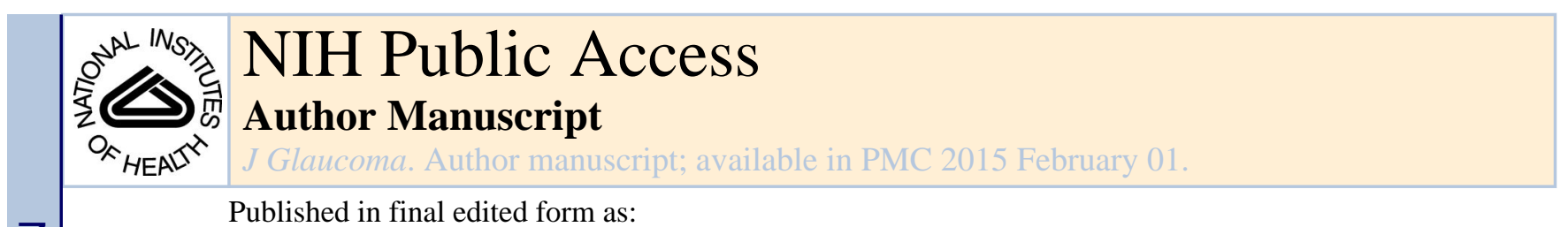

Published in final edited form as:

J Glaucoma. 2015 February ; 24(2): 117-121. doi:10.1097/IJG.0b013e31829d9bb0.

\title{
Differences in Ocular Blood Flow in Glaucoma Between Patients of African and European Descent
}

\author{
Brent Siesky, PhD ${ }^{\star}$, Alon Harris, PhD, FARVO ${ }^{\star}$, Lyne Racette, PhD”, Rania Abassi, MD*, \\ Kaarthik Chandrasekhar, MD*, Leslie A. Tobe, MD*, Jennifer Behzadi, MD*, George Eckert, \\ MAS $^{\dagger}$, Annahita Amireskandari, MD*, and Michael Muchnik, BS, MS ${ }^{*}$ \\ *Departments of Ophthalmology, Eugene and Marilyn Glick Eye Institute, Indiana University \\ School of Medicine, Indianapolis, IN \\ †Biostatistics, Indiana University School of Medicine, Indianapolis, IN
}

\section{Abstract}

Purpose-To investigate differences in ocular blood flow in individuals of African descent (AD) and European descent (ED) with open angle glaucoma (OAG).

\begin{abstract}
Patients and Methods-A retrospective data analysis was performed on OAG patients of AD and ED who were previously examined for ocular blood flow within the Department of Ophthalmology at Indiana University School of Medicine. Data analysis included blood pressure, heart rate, visual fields, intraocular pressure, ocular perfusion pressure, and color Doppler imaging of retrobulbar vessels. Color Doppler imaging measurements were performed on ophthalmic, central retinal, and nasal and temporal short posterior ciliary arteries, with peak systolic (PSV) and end diastolic velocities (EDV) as well as the Pourcelot vascular resistive index calculated for each vessel. Two-sample $t$ tests of unequal variance were performed with $P$ values $<0.05$ considered statistically significant.
\end{abstract}

Results-OAG patients of AD had statistically significant lower retrobulbar blood flow values than patients of ED including lower ophthalmic artery PSV ( $P=0.0001)$, ophthalmic artery EDV $(P=0.0008)$, central retinal artery PSV $(P=0.01)$, temporal short posterior ciliary artery PSV $(P=0.0037)$, and nasal short posterior ciliary artery PSV $(P<0.0001)$. No significant differences were found in terms of intraocular pressure or visual field parameters.

Conclusions-Significantly lower blood flow values were identified in all retrobulbar blood vessels in AD compared with ED OAG patients. These findings suggest that the contribution of ocular blood flow to the disease process may be different in AD compared with ED OAG patients.

\section{Keywords}

glaucoma; blood flow; African descent; race

Copyright $(92013$ by Lippincott Williams \& Wilkins

Reprints: Alon Harris, PhD, FARVO, Department of Ophthalmology, Eugene and Marilyn Glick Eye Institute, Indiana University School of Medicine, 1160 W. Michigan St., Indianapolis, IN 46202 (alharris@ indiana.edu).

Disclosure: The authors declare no conflict of interest. 
Glaucoma, a multifactorial optic neuropathy characterized by progressive retinal ganglion cell death and visual field loss, is a leading cause of blindness in persons of African descent (AD). ${ }^{1-3}$ Glaucoma affects up to 6 times as many persons of $\mathrm{AD}$ as it does those of European descent (ED). ${ }^{1,2}$ In addition to their increased risk of developing glaucoma, persons of $\mathrm{AD}$ tend to develop open angle glaucoma (OAG) earlier, have higher disease severity, and experience glaucoma progression more quickly, resulting in greater loss of visual function. ${ }^{4-10}$ Although differences exist in the development and progression rates of OAG between persons of $\mathrm{AD}$ and $\mathrm{ED}$, the mechanisms behind this disparity have yet to be elucidated.

Multiple studies have addressed factors that may play a role in the development of glaucoma. A recent study suggested that there are no significant differences in the aqueous humor dynamics between patients of AD and ED. ${ }^{11}$ Several studies have shown that people of AD have significantly thinner corneas, higher intraocular pressure (IOP), larger optic discs, greater optic disc area, and deeper maximum cup depth. ${ }^{5,12}$ Furthermore, increased levels of oxygen in the anterior chamber have been found in individuals of AD as compared with those of ED possibly leading to increased oxidative stress and damage. ${ }^{13}$

Despite being a leading cause of impaired vision worldwide, the risk factors for OAG are not fully understood. IOP is the only currently treatable risk factor for OAG, although a high percentage of individuals with elevated IOP do not develop glaucoma. ${ }^{14}$ In addition, many patients experience glaucoma progression despite meeting target IOP, suggesting other contributing factors in OAG disease onset and progression. ${ }^{15,16}$

Many clinical studies have linked abnormalities in ocular perfusion pressure (OPP) and blood flow to glaucomatous optic neuropathy. Blood flow deficiencies of the retinal, choroid, and retrobulbar circulations have been reported in patients with OAG. ${ }^{16}$ In large population-based trials, reduced OPP has been linked to the prevalence, incidence, and progression of glaucoma. ${ }^{17-21}$ Systemic and local vascular abnormalities have also been linked to OAG including arterial hypertension, nocturnal hypotension, optic disc hemorrhage, migraine, and aging of the vasculature. ${ }^{16,21}$ Although some research has indicated vascular parameters are associated with glaucoma, their exact relationship to glaucoma progression remains insufficiently investigated.

In addition to experiencing disparity in $\mathrm{OAG}$, persons of $\mathrm{AD}$ tend to have more systemic vasculature complications, leading to numerous medical conditions. Persons of AD have considerably higher rates of cardiovascular disease (CVD) with early onset and high severity, significantly affected by risk factors such as obesity, smoking, and a sedentary lifestyle. ${ }^{22}$ According to the American Heart Association, the death rate per 100,000 persons for stroke were 41.7 for white males, 67.1 for black males, 41.1 for white females, and 57.0 for black females. ${ }^{23}$ In the African Descent and Glaucoma Evaluation Study, those of AD were documented as having a higher percentage of reported diabetes mellitus, higher blood pressure, and higher IOP. ${ }^{24}$ Nagasubramanian and Weale ${ }^{25}$ studied the vasculature of the optic disc between normal and glaucomatous whites, Africans, and Afro-Caribbeans. The vessel pattern differed distinctly between the ethnic groups, with white rims showing a higher number of smaller vessels as well as capillary crossings. 
Such studies have demonstrated that there could be a relationship between poor vasculature and the severity and earlier onset of glaucoma in AD populations. To the best of our knowledge, this is the first investigation that directly examines ocular blood flow parameters between persons of $\mathrm{AD}$ and $\mathrm{ED}$ with $\mathrm{OAG}$ in an attempt to help explain the mechanisms behind the racial disparities in glaucoma.

\section{MATERIALS AND METHODS}

A retrospective analysis was performed using prospectively collected data from previously examined OAG patients within the Department of Ophthalmology at Indiana University School of Medicine. All participants were established OAG patients and had prior visual field experience within the clinic where all measurements used in this analysis were performed. Data from only 1 qualified eye was used for each measurement. Data from AD and ED OAG patients were separated based on self-reported race and analyzed. All patients had OAG based on a clinical examination, visual acuity of 20/60 or better, had treatment of timolol (only) for 4 weeks while undergoing washout of all other glaucoma treatments to ensure treatment uniformity. Standard Automated Perimetry was performed using the 24-2 program and Swedish Interactive Thresholding Algorithm on the Humphrey Field Analyzer (Carl Zeiss Meditec, Dublin, CA). To be included in the study, the clinical diagnosis of OAG had to be confirmed in the study eye by a fellowship-trained glaucoma specialist based upon criteria representative of glaucomatous optic disc or retinal nerve fiber layer structural abnormalities and/or visual field damage consistent with glaucomatous damage.

All participants were free from other ocular disease or trauma, avoided caffeine for 2 hours before exams, and had stable blood pressure. All measurements were performed by a single operator (B.S.) in the same clinic between the hours of 8:00 AM and 4:00 PM.

IOP was obtained using Goldmann applanation tonometry. Brachial artery systolic and diastolic blood pressure and pulse were assessed after a 5-minute rest period using a calibrated automated sphygmomanometer at the beginning of each study visit. Mean arterial pressure was calculated as $2 / 3$ diastolic blood pressure $+1 / 3$ systolic blood pressure. OPPs were calculated as $2 / 3$ mean arterial pressure - IOP.

Color Doppler imaging (CDI) of the retrobulbar blood vessels was conducted using Philips HDI 5000 SonoCT Ultrasound System with the microvascular small parts clinical option (Philips Medical Systems, Bothell, WA) using a 7.5MHz linear probe as described in detail previously. ${ }^{26} \mathrm{CDI}$ measurements were taken in the ophthalmic (OA), central retinal (CRA), and nasal and temporal (NPCA/TPCA) short posterior ciliary arteries. In each vessel, peak systolic (PSV) and end diastolic (EDV) velocities were determined and the Pourcelot vascular resistive index (RI) was calculated [RI=(PSV - EDV)/PSV]. These techniques have been previously shown to yield reproducible measurements of retrobulbar blood flow velocities and peripheral vascular resistance. ${ }^{26}$

For each subject, all available data were included for all measurements. Two-sample $t$ tests of unequal variance were performed, and all available variables for all patients were included with $P$ values $<0.05$ considered as statistically significant. 


\section{RESULTS}

Table 1 lists the mean values, SD, $P$ values, and number of patients for visual fields, IOP, blood pressure, heart rate, and OPP in each study group. The mean age and sex of the subjects were [age: 68.5 (10.2), 52\% female for AD and 70.2 (11.4), 58\% female for ED]. There were no significant differences between AD and ED OAG patients in visual field mean deviation or pattern SD. Also, no statistically significant differences were found in terms of IOP between groups. Persons of AD had a significantly higher heart rate $(P=0.0065)$, systolic blood pressure $(P=0.0001)$, and diastolic blood pressure $(P=0.0022)$ than persons of ED. OPP was significantly higher in OAG patients of AD compared with $\mathrm{ED}(P=0.002)$.

OAG patients of AD had statistically significant lower retrobulbar blood flow values than ED patients. Significantly lower retrobulbar blood flow values for OAG patients of AD compared with ED were found for the following retrobulbar blood flow parameters: OA PSV $(P=0.0001)$, OA EDV $(P=0.0008)$, CRA PSV $(P=0.010)$, NPCA PSV $(P<0.0001)$, and TPCA PSV $(P=0.0037)$. Only 1 CDI variable was worse in ED with a higher NPCA RI $(P=0.0029)$, whereas all other CDI variables were not significantly different between groups as shown in Table 2 .

\section{DISCUSSION}

The mechanism behind the differences in OAG incidence, prevalence, and progression between $\mathrm{AD}$ and $\mathrm{ED}$ populations has yet to be elucidated. Glaucoma risk factors continue to evolve away from an IOP-only disease to a multifactorial optic neuropathy. Reductions in OPP and blood flow have consistently been shown to be involved in the OAG disease process, although the differences in their contribution to OAG pathology between persons of $\mathrm{AD}$ and $\mathrm{ED}$ have yet to be defined. Persons of $\mathrm{AD}$ are known to have higher incidence of hypertension and other systemic vascular diseases. This provides a strong rationale to investigate possible differences that may exist between persons of AD and ED in OPP and blood flow.

The results of this study show that persons of AD with OAG had significantly higher systolic and diastolic blood pressures than their ED counterparts. This is consistent with previous studies demonstrating a greater incidence of hypertension in $\mathrm{AD}$ populations. $5,27,28$ Prior studies have also noted increased prevalence of diabetes along with hypertension in persons of AD. ${ }^{5,27,28}$ In our analysis, OAG patients of AD also demonstrated significantly higher heart rates than persons of ED. This may be related to the increased prevalence of hypertension and diabetes in this population. An increased heart rate, in addition to hypertension and diabetes, is considered a risk factor for CVD. ${ }^{29,30}$ Results from the Ocular Hypertension Treatment Study suggest that a greater occurrence of CVD may be linked to the higher incidence of glaucoma in African Americans. ${ }^{31}$

Some studies attribute the increased prevalence and incidence of OAG in persons of AD to elevated IOP. For example, it has been shown that the risk of developing glaucoma increases 25 -fold between an IOP of 17 and $25 \mathrm{~mm} \mathrm{Hg} .{ }^{20}$ The Barbados Eye Study demonstrated that 
African Americans examined had much higher IOP levels. ${ }^{32}$ In the present study, we do not believe that the racial differences we observed in ocular blood can be explained by differences in IOP because we found no differences in treated IOP between the AD and ED groups. It is worth noting that analyses from several population-based studies, including the Barbados, Rotterdam, and Baltimore studies revealed that increased IOP may be linked with hypertension. 5,27,28,33,34 Hence, the high incidence of hypertension found in the AD population may account for some of the reports of higher IOP. ${ }^{5,27,28}$ Furthermore the racial differences in ocular blood flow that we report here were likely not due to differences in the severity of glaucoma between the 2 groups. Indeed, we found no differences in the visual parameters between people of $\mathrm{AD}$ and $\mathrm{ED}$, although the number of available fields for $\mathrm{OAG}$ patients of $\mathrm{AD}$ was less than those available for $\mathrm{OAG}$ of $\mathrm{ED}$ which is a limitation.

The comparison of retrobulbar blood flow parameters between AD and ED OAG patients revealed a uniform reduction in retrobulbar circulation in persons of AD compared with their ED counterparts. In our study, OA PSV and EDV, CRA PSV, NPCA PSV, and TPCA PSV were significantly lower in persons of AD compared with ED OAG patients. With only 1 CDI variable was worse in ED (higher NPCA RI), our data indicate a clear trend of lower ocular circulation in persons of AD compared with ED despite similar IOP and visual field defects. Previous studies have demonstrated reduced blood flow velocities in the retrobulbar vessels of glaucoma patients. ${ }^{35-39}$ Specifically, Martinez and Sanchez ${ }^{40}$ showed that vascular resistance of retrobulbar vessels may predict visual field progression, whereas Galassi et al ${ }^{41}$ demonstrated that constricted retrobulbar flow was associated with visual field loss. These findings suggest that the contribution of ocular blood flow to the disease process may be different in AD compared with ED OAG patients.

An increase in IOP or reduction in systemic blood pressure has the potential to lower retrobulbar blood flow values through a reduction in OPP. ${ }^{42,43}$ However, the lower blood flow values we found in all retrobulbar blood vessels in patients of $A D$ are not explained by IOP or systemic blood pressure, as no difference in IOP was found between the groups and blood pressure was higher in patients of $\mathrm{AD}$. This is a strong indication that reduced localized ocular circulation may contribute to OAG differently in persons of AD compared with persons of ED. Reductions in retrobulbar blood flow velocities in persons of AD may represent non-IOP and OPP-related localized vascular deficits, faulty vascular regulation, or may be a part of an overall systemic vascular disease process apparent in the $\mathrm{AD}$ population. It is also important to consider that IOP may not be an ideal surrogate of the resistance of ocular perfusion. The resistance from ocular vessel may play a more important role in ocular perfusion.

Our study has several limitations to acknowledge. These include a lack of prospective follow-up and non-availability of optic disc parameters such as cup-disc ratio (C/D ratio), corneal thickness, oxygen levels, and genetics. For example, several studies have shown that persons of $\mathrm{AD}$ have significantly larger $\mathrm{C} / \mathrm{D}$ ratios than whites. ${ }^{7,31,44-46} \mathrm{C} / \mathrm{D}$ ratio, like diabetes and hypertension, has been positively linked with IOP. ${ }^{32}$ Race was self-reported, which may also be a limitation of the study. However, self-described race has demonstrated a high correlation with more sophisticated measures of racial classification using genetic admixture techniques and thus is likely an adequate surrogate measure. ${ }^{10,47}$ Our study is 
also limited by the fact that there was a greater number of patients of ED than AD which may affect the comparisons between groups, although all available data for all subjects were included for each measure without selection bias. As our data were retrospective and deidentified at the time of analysis we also could not perform more advanced statistical analysis such as multivariate or multiple linear regressions including all the potential-related variables to screen the independent associated factors. It is important to note, however, that the direction of the results we obtained suggests that the differences would be in the same direction and likely stronger even after adjusting for possible confounds such as blood pressure, heart rate, and OPP. Indeed, people of AD had higher blood pressure, heart rate, and OPP, yet lower ocular blood flow. This remains a limitation of the current data set nonetheless. It is also important to note that our study, while suggestive, is not able to establish causality of OAG due to lower ocular blood flow due to its retrospective design.

This study, to the best of our knowledge, is the first to demonstrate directly measured differences in ocular blood flow between AD and ED OAG patients. Our study revealed a higher incidence of cardiovascular risk factors, which have been linked to glaucoma, such as hypertension and increased heart rate, in persons with OAG of AD compared with ED patients. Significantly lower blood flow values were identified in all retrobulbar blood vessels in persons of AD compared with ED OAG patients despite similar IOP and visual field parameters. These findings suggest that the contribution of ocular blood flow to glaucoma may be different in AD compared with ED patients. Additional research is needed to determine whether the differences in ocular blood flow reported in this study may be related to disparities in the rate of glaucoma progression.

\section{Acknowledgments}

Supported in part by an unrestricted grant from Research to Prevent Blindness, New York, NY.

\section{References}

1. Congdon N, O'Colmain B, Klaver CC, et al. Causes and prevalence of visual impairment among adults in the United States. Arch Ophthalmol. 2004; 122:477-485. [PubMed: 15078664]

2. Friedman DS, Wolfs RC, O'Colmain BJ, et al. Prevalence of open-angle glaucoma among adults in the United States. Arch Ophthalmol. 2004; 122:532-538. [PubMed: 15078671]

3. Hyman L, Wu SY, Connell AM, et al. Prevalence and causes of visual impairment in the Barbados Eye Study. Ophthalmology. 2001; 108:1751-1756. [PubMed: 11581045]

4. Wilson R, Richardson TM, Hertzmark E, et al. Race as a risk factor for progressive glaucomatous damage. Ann Ophthalmol. 1985; 17:653-659. [PubMed: 4073724]

5. Racette L, Wilson MR, Zangwill LM, et al. Primary open-angle glaucoma in blacks: a review. Surv Ophthalmol. 2003; 48:295-313. [PubMed: 12745004]

6. Sommer A, Tielsch JM, Katz J, et al. Racial differences in the cause-specific prevalence of blindness in east Baltimore. N Engl J Med. 1991; 325:1412-1417. [PubMed: 1922252]

7. Martin MJ, Sommer A, Gold EB, et al. Race and primary open-angle glaucoma. Am J Ophthalmol. 1985; 99:383-387. [PubMed: 3985075]

8. Wilensky JT, Gandhi N, Pan T. Racial influences in open-angle glaucoma. Ann Ophthalmol. 1978; 10:1398-1402. [PubMed: 718042]

9. Racette L, Liebmann JM, Girkin CA, et al. African Descent and Glaucoma Evaluation Study (ADAGES): III. Ancestry differences in visual function in healthy eyes. Arch Ophthalmol. 2010; 128:551-559. [PubMed: 20457975] 
10. Tielsch JM, Sommer A, Katz J, et al. Racial variations in the prevalence of primary open-angle glaucoma. The Baltimore Eye Survey. JAMA. 1991; 266:369-374. [PubMed: 2056646]

11. Beltran-Agullo L, Alaghband P, Rashid S, et al. Comparative human aqueous dynamics study between black and white subjects with glaucoma. Invest Ophthalmol Vis Sci. 2011; 52:94259430. [PubMed: 21980001]

12. Girkin CA, McGwin G Jr, Xie A, et al. Differences in optic disc topography between black and white normal subjects. Ophthalmology. 2005; 112:33-39. [PubMed: 15629817]

13. Hollows RC, Graham PA. Intraocular pressure, glaucoma, and glaucoma suspects in a defined population. Br J Ophthalmol. 1996; 50:570-577. [PubMed: 5954089]

14. Heijl A, Leske MC, Bengtsson B, et al. Reduction of intraocular pressure and glaucoma progression: results from the Early Manifest Glaucoma Trial. Arch Ophthalmol. 2002; 120:12681279. [PubMed: 12365904]

15. Collaborative Normal-Tension Glaucoma Study Group. Comparison of glaucomatous progression between untreated patients with normal-tension glaucoma and patients with therapeutically reduced intraocular pressure. Am J Ophthalmol. 1998; 126:487-497. [PubMed: 9780093]

16. Harris A, Kagemann L, Ehrlich R, et al. Measuring and interpreting ocular blood flow and metabolism in glaucoma. Can J Ophthalmol. 2008; 43:328-336. [PubMed: 18443609]

17. Tielsch JM, Katz J, Sommer A, et al. Hypertension, perfusion pressure, and primary open-angle glaucoma. A population-based assessment. Arch Ophthalmol. 1995; 113:216-221. [PubMed: 7864755]

18. Quigley HA, West SK, Rodriguez J, et al. The prevalence of glaucoma in a population-based study of Hispanic subjects: Proyecto VER. Arch Ophthalmol. 2001; 119:1819-1826. [PubMed: 11735794]

19. Bonomi L, Marchini G, Marrafa M, et al. Vascular risk factors for primary open angle glaucoma: the Egna-Neumarkt Study. Ophthalmology. 2000; 107:1287-1293. [PubMed: 10889099]

20. Leske MC, Wu SY, Nemesure B, et al. Incident open-angle glaucoma and blood pressure. Arch Ophthalmol. 2002; 120:954-959. [PubMed: 12096967]

21. Moore D, Harris A, Wudunn D, et al. Dysfunctional regulation of ocular blood flow: a risk factor for glaucoma? Clin Ophthalmol. 2008; 2:849-861. [PubMed: 19668439]

22. Ferdinand KC. Cardiovascular disease in blacks: can we stop the clock? J Clin Hypertens (Greenwich). 2008; 10:382-389. [PubMed: 18453798]

23. [Accessed July 8, 2010] Stroke Statistics. Available at: http://www.heart.org/HEARTORG/ General/Heart-and-Stroke-Association-Statistics_UCM_319064_SubHomePage.jsp

24. Sample PA, Girkin CA, Zangwill LM, et al. The African Descent and Glaucoma Evaluation Study (ADAGES): design and baseline data. Arch Ophthalmol. 2009; 127:1136-1145. [PubMed: 19752422]

25. Nagasubramanian S, Weale RA. Ethnic variability of the vasculature of the optic disc in normal and in glaucomatous eyes. Eur J Ophthalmol. 2004; 14:501-507. [PubMed: 15638099]

26. Williamson TH, Harris A. Color Doppler ultrasound imaging of the eye and orbit. Surv Ophthalmol. 1996; 40:255-267. [PubMed: 8658337]

27. Hennis A, Wu SY, Nemesure B, et al. Hypertension, diabetes, and longitudinal changes in intraocular pressure. Ophthalmology. 2003; 110:908-914. [PubMed: 12750088]

28. Wu SY, Leske MC. Associations with intraocular pressure in the Barbados Eye Study. Arch Ophthalmol. 1997; 115:1572-1576. [PubMed: 9400792]

29. Chambless LE, Folsom AR, Sharrett AR, et al. Coronary heart disease risk prediction in the Atherosclerosis Risk in Communities (ARIC) study. J Clin Epidemiol. 2003; 56:880-890. [PubMed: 14505774]

30. McGruder HF, Malarcher AM, Antoine TL, et al. Racial and ethnic disparities in cardiovascular risk factors among stroke survivors: United States 1999 to 2001. Stroke. 2004; 35:1557-1561. [PubMed: 15192252]

31. Higginbotham EJ, Gordon MO, Beiser JA, et al. The Ocular Hypertension Treatment Study: topical medication delays or prevents primary open-angle glaucoma in African American individuals. Arch Ophthalmol. 2004; 122:813-820. [PubMed: 15197055] 
32. Leske MC, Connell AM, Wu SY, et al. Distribution of intraocular pressure: the Barbados Eye Study. Arch Ophthalmol. 1997; 115:1051-1057. [PubMed: 9258228]

33. Dielemans I, de Jong PT, Stolk R, et al. Primary open-angle glaucoma, intraocular pressure, and diabetes mellitus in the general elderly population. The Rotterdam Study. Ophthalmology. 1996; 103:1271-1275. [PubMed: 8764798]

34. Tielsch JM, Katz J, Quigley HA, et al. Diabetes, intraocular pressure, and primary open-angle glaucoma in the Baltimore Eye Survey. Ophthalmology. 1995; 102:48-53. [PubMed: 7831041]

35. Akarsu C, Bilgili MY. Color Doppler imaging in ocular hypertension and open-angle glaucoma. Graefes Arch Clin Exp Ophthalmol. 2004; 242:125-129. [PubMed: 14663592]

36. Chiou HJ, Chou YH, Liu CJ, et al. Evaluation of ocular arterial changes in glaucoma with color Doppler ultrasonography. J Ultrasound Med. 1999; 18:295-302. [PubMed: 10206218]

37. Nicolela MT, Walman BE, Buckley AR, et al. Ocular hypertension and primary open-angle glaucoma: a comparative study of their retrobulbar blood flow velocity. J Glaucoma. 1996; 5:308310. [PubMed: 8897229]

38. Sergott RC, Aburn NS, Trible JR, et al. Color Doppler imaging: methodology and preliminary results in glaucoma. Surv Ophthalmol. 1994; 38:S65-S71. [PubMed: 7940149]

39. Ustymowicz A, Krejza J, Tarasow E, et al. Blood flow parameters in ocular vessels of patients with glaucoma. Klin Oczna. 1999; 101:445-449. [PubMed: 10786053]

40. Martinez A, Sanchez M. Predictive value of colour Doppler imaging in a prospective study of visual field progression in primary open-angle glaucoma. Acta Ophthalmol Scand. 2005; 83:716722. [PubMed: 16396650]

41. Galassi F, Sodi A, Ucci F, et al. Ocular hemodynamics and glaucoma prognosis: a color Doppler imaging study. Arch Ophthalmol. 2003; 121:1711-1715. [PubMed: 14662590]

42. Gherghel D, Orgul S, Gugleta K, et al. Relationship between ocular perfusion pressure and retrobulbar blood flow in patients with glaucoma with progressive damage. Am J Ophthalmol. 2000; 130:597-605. [PubMed: 11078838]

43. Harris A, Kagemann L, Cioffi GA. Assessment of human ocular hemodynamics. Surv Ophthalmol. 1998; 42:509-533. [PubMed: 9635901]

44. Beck RW, Messner DK, Musch DC, et al. Is there a racial difference in physiologic cup size? Ophthalmology. 1985; 92:873-876. [PubMed: 4022570]

45. Chi T, Ritch R, Stickler D, et al. Racial differences in optic nerve head parameters. Arch Ophthalmol. 1989; 107:836-839. [PubMed: 2730402]

46. Zangwill LM, Weinreb RN, Berry CC, et al. Racial differences in optic disc topography: baseline results from the confocal scanning laser ophthalmoscopy ancillary study to the Ocular Hypertension Treatment Study. Arch Ophthalmol. 2004; 122:22-28. [PubMed: 14718290]

47. Rosenberg NA, Pritchard JK, Weber JL, et al. Genetic structure of human populations. Science. 2002; 298:2381-2385. [PubMed: 12493913] 


\section{TABLE 1}

The Visual Field, Intraocular Pressure, Blood Pressure, Heart Rate, and Ocular Perfusion Pressure Parameters are Presented for Each Study Group

\begin{tabular}{lccl}
\hline & AD Mean \pm SD (n) & ED Mean \pm SD (n) & $P$ \\
\hline MD (dB) & $-1.94 \pm 2.77(19)$ & $-2.17 \pm 4.38(74)$ & 0.78 \\
PSD (dB) & $3.02 \pm 2.55(19)$ & $3.23 \pm 3.68(74)$ & 0.77 \\
IOP (mm Hg) & $15.99 \pm 4.03(60)$ & $15.50 \pm 4.44(115)$ & 0.46 \\
Systolic BP (mm Hg) & $137.45 \pm 19.66(66)$ & $126.59 \pm 14.03(123)$ & $\mathbf{0 . 0 0 0 1}$ \\
Diastolic BP (mm Hg) & $83.17 \pm 11.53(66)$ & $77.97 \pm 9.57(123)$ & $\mathbf{0 . 0 0 2 2}$ \\
HR (bpm) & $78.68 \pm 18.01(66)$ & $71.92 \pm 11.01(123)$ & $\mathbf{0 . 0 0 6 5}$ \\
Mean OPP (mm Hg) & $51.42 \pm 8.98(60)$ & $47.14 \pm 7.54(115)$ & $\mathbf{0 . 0 0 2 1}$ \\
\hline
\end{tabular}

Bold $P$-values indicate statistical significance.

$\mathrm{AD}$ indicates African descent; $\mathrm{BP}$, blood pressure; ED, European descent; HR, heart rate; IOP, intraocular pressure; MD, mean deviation; n, number of eyes; OPP, ocular perfusion pressure; PSD, pattern SD. 


\section{TABLE 2}

The Ocular Blood Flow Parameters From Color Doppler Imaging are Presented for Each Study Group

\begin{tabular}{lccc}
\hline & AD Mean \pm SD $(\mathbf{n})$ & ED Mean \pm SD $(\mathbf{n})$ & $\boldsymbol{P}$ \\
\hline OA-PSV $(\mathrm{cm} / \mathrm{s})$ & $26.51 \pm 6.19(61)$ & $30.90 \pm 8.21(121)$ & $\mathbf{0 . 0 0 0 1}$ \\
OA-EDV $(\mathrm{cm} / \mathrm{s})$ & $6.92 \pm 1.99(62)$ & $8.17 \pm 2.90(121)$ & $\mathbf{0 . 0 0 0 8}$ \\
OA-RI & $0.73 \pm 0.056(63)$ & $0.73 \pm 0.070(138)$ & 0.89 \\
CRA-PSV $(\mathrm{cm} / \mathrm{s})$ & $7.11 \pm 1.87(62)$ & $8.07 \pm 3.12(121)$ & $\mathbf{0 . 0 1 0}$ \\
CRA-EDV $(\mathrm{cm} / \mathrm{s})$ & $1.80 \pm 0.70(65)$ & $1.89 \pm 0.77(139)$ & 0.40 \\
CRA-RI & $0.75 \pm 0.058(62)$ & $0.76 \pm 0.064(121)$ & 0.053 \\
TPCA-PSV $(\mathrm{cm} / \mathrm{s})$ & $5.36 \pm 1.35(48)$ & $6.08 \pm 1.35(95)$ & $\mathbf{0 . 0 0 3 7}$ \\
TPCA-EDV $(\mathrm{cm} / \mathrm{s})$ & $1.78 \pm 0.70(48)$ & $1.83 \pm 0.57(100)$ & 0.71 \\
TPCA-RI & $0.66 \pm 0.097(47)$ & $0.70 \pm 0.073(94)$ & 0.052 \\
NPCA-PSV $(\mathrm{cm} / \mathrm{s})$ & $5.44 \pm 1.37(47)$ & $6.51 \pm 1.46(99)$ & $<\mathbf{0 . 0 0 0 1}$ \\
NPCA-EDV $(\mathrm{cm} / \mathrm{s})$ & $1.79 \pm 0.56(49)$ & $1.88 \pm 0.61(110)$ & 0.37 \\
NPCA-RI & $0.67 \pm 0.079(47)$ & $0.71 \pm 0.074(99)$ & $\mathbf{0 . 0 0 2 9}$ \\
\hline
\end{tabular}

Bold $P$-values indicate statistical significance.

AD indicates African descent; CRA, central retinal artery; ED, European descent; EDV, end diastolic velocity; NPCA, nasal posterior ciliary artery; OA, ophthalmic artery; PSV, peak systolic velocity; RI, Pourcelot vascular resistive index; TPCA, temporal posterior ciliary artery. 